\title{
First laboratory results from FOURIER, the initial science combiner at the MROI
}

\author{
Daniel J. Mortimer ${ }^{\mathrm{a}}$, David F. Buscher ${ }^{\mathrm{a}}$, Michelle J. Creech-Eakman ${ }^{\mathrm{b}}$, Chris Haniffa, Edgar \\ Ligon $^{\mathrm{b}}$, James Luis ${ }^{\mathrm{a}}$, Christopher Salcido ${ }^{\mathrm{b}}$, Eugene Seneta ${ }^{\mathrm{a}}$, Xiaowei Sun ${ }^{\mathrm{a}}$, and John Young ${ }^{\mathrm{a}}$ \\ ${ }^{a}$ Cambridge University, Cavendish Laboratory, JJ Thomson Ave, Cambridge, UK \\ ${ }^{b}$ Magdalena Ridge Observatory/New Mexico Tech, 801 Leroy Place, Socorro, NM, USA
}

\begin{abstract}
We present the design and testing of FOURIER, the first generation science beam combiner for the MROI. FOURIER is a three-way, J, $\mathrm{H}$ and $\mathrm{K}$ band image plane combiner which is designed primarily for observations at faint limiting magnitudes. We outline the main science requirements and discuss how the design of FOURIER contributes to meeting these requirements. We present the first laboratory characterisation of the instrument including validation of the PSF profile, demonstration of high contrast fringes, and the spectral resolution of the instrument, all of which show promising results. We conclude by discussing the path to deployment of FOURIER at the MROI ahead of the first science observation at the array.
\end{abstract}

Keywords: Interferometry, Beam Combiner, MROI, spectro-interferometry, J-Band, H-Band, K-Band

\section{INTRODUCTION}

The Magdalena Ridge Observatory Interferometer (MROI) is a next generation long baseline optical interferometer under development by a collaboration between New Mexico Tech and the University of Cambridge. When completed it will be a 10-telescope array with 28 Unit Telescope (UT) stations arranged in a equilateral Y shape operating on baselines between 7.8-347 metres, and will conduct observations in both the visible and near-infrared $(\lambda=0.6 \mu \mathrm{m}-2.4 \mu \mathrm{m})$. The project is currently progressing towards operating three telescopes in the near-infrared. The first telescope has recently been deployed on the array with starlight being subsequently carried through the entire beam train of one arm and observed in the Inner-Beam Combining Area (I-BCA) for the first time.

To support the project as it moves towards operating three telescopes, the Free-space Optical multi-apertUre combineR for IntERferometry (FOURIER) is under construction. FOURIER is a three-way, cryogenically cooled and spectrally dispersed image plane science beam combiner and will operate in the $\mathrm{J}, \mathrm{H}$ and $\mathrm{K}$ bands $(\lambda=1.1 \mu \mathrm{m}-$ $2.4 \mathrm{~mm}$ ). It will perform the first closure phase measurements at the array, conduct early science during the three telescope phase of the project as well as assist in demonstrating the baseline bootstrapping capabilities of the MROI. ${ }^{1}$

The final design review for FOURIER was held in December 2019 and the instrument is currently undergoing room temperature (warm) laboratory testing at the University of Cambridge. Once these tests are complete the cold optics and detector will be integrated with the liquid nitrogen cooled cryostat for further laboratory tests and subsequently delivered to the observatory for commissioning in the third or fourth quarter of 2021.

Send correspondence to D.J.M.

D.J.M.: E-mail: djm244@cam.ac.uk 


\subsection{Scientific Motivation}

The MROI is designed to push the boundaries of what is possible with long baseline interferometry in two key areas. Firstly by increasing the complexity of images that can be reconstructed (by increasing the number of telescopes and hence baselines available). Secondly by reaching limiting magnitudes several magnitudes fainter than current generation arrays, enabling observations of many more targets for a given class of objects. ${ }^{2}$ The $^{3}$ scientific motivations for FOURIER are discussed in more detail in Mortimer \& Buscher ${ }^{3}$ and are summarised in the following paragraphs.

FOURIER will initially have access to a limited number of $10 \mathrm{~m}$ class baselines, limiting the spatial resolution and UV plane sampling. Instead we will take advantage of both the significant increase in limiting magnitude FOURIER will reach over current generation arrays, as well as its ability to conduct observations across multiple spectral bands covering a large range of wavelengths. As a result early science will focus on targets which do not require high spatial resolutions, examples of which would be binary systems or giant stars, similar to the work of Weigelt et al. ${ }^{4}$

As longer baselines become available new science which benefits from the unique combination of high spatial resolution, extensive UV coverage and faint limiting magnitudes will be conducted. A top priority for the observatory is Active Galactic Nuclei (AGN) science. Here the MROI aims to characterise the spatial morphology of an unprecedented 100 AGN to answer questions such as: what is the frequency of occurrence of tori around AGN; and are the observed morphologies consistent with 'unified' schemes. ${ }^{5}$ Young stellar object (YSO) science will also benefit from the unique capabilities of the MROI. The array will afford access to a greater number of targets than are currently observable via optical interferometry, as well as significantly improve the ability to image the complex structure within the disks around YSOs, providing a census of disk properties. ${ }^{2}$

\section{DESIGN}

\subsection{Design Approach}

The key science-motivated design requirements for FOURIER are the following:

- Under typical best seeing conditions at the site of $r_{0}=14 \mathrm{~cm}$ and $t_{0}=4.4 \mathrm{~ms}$ (defined at $\lambda=500 \mathrm{~nm}$, where $r_{0}$ and $t_{0}$ are the Fried parameter and coherence time respectively), the Signal to Noise Ratio (SNR) of the fringe power spectrum in a single spectral channel shall be greater than 2 within 5 minutes of incoherent integration when observing an unresolved $10^{\text {th }}$ magnitude object, with a goal of reaching this SNR for an unresolved $11^{\text {th }}$ magnitude object.

- The instrument shall form fringes over the whole of a single near-infrared band (one of $\mathrm{J}, \mathrm{H}$ or $\mathrm{K}$ ) simultaneously. The band shall be selectable between $\mathrm{J}, \mathrm{H}$ and $\mathrm{K}$ by reconfiguration of the instrument.

- It must support spectro-interferometry with a minimum resolving power of $R=70$ in all bands.

- The instrument shall be designed to allow operation with 3 beams for closure phase measurements, and shall allow operation with 2 beams when fewer telescopes are available.

In addition to the design goals stated above, there were two constraints which had a large impact on the design of the instrument. The first was the rapid development time frame from conceptual design to on-sky commissioning within four years, to begin science as soon as possible after first fringes at the MROI, the second was to develop the instrument on a low budget.

In conjunction with the limiting magnitude requirement, these constraints pointed towards a design using as few optical components as possible. This not only reduces technical risk, but also improves the throughput by minimising the number of optical surfaces. The final design has only six optical components including the spatial filter and no motorised mechanics. 


\subsection{Optical Design}

The optical design of FOURIER is presented in figure 1. The three input beams of diameter $\mathrm{d}=13 \mathrm{~mm}$ are arranged with non-redundant spacings of $2 \mathrm{~d}, 4 \mathrm{~d}$ and $6 \mathrm{~d}$. The beams travel right to left initially where they are reflected into the cryostat by the 6 inch spherical mirror (M1) and are reflected through the cryostat window to the cold optics. Here they are first spatially filtered along the spectral axis by a slit placed at the focal point of M1. The beams are then re-imaged along two perpendicular axes by M2 and L1, being spectrally dispersed by the prism between the two. The dispersed interference fringes are then recorded on the SAPHIRA detector. ${ }^{6}$

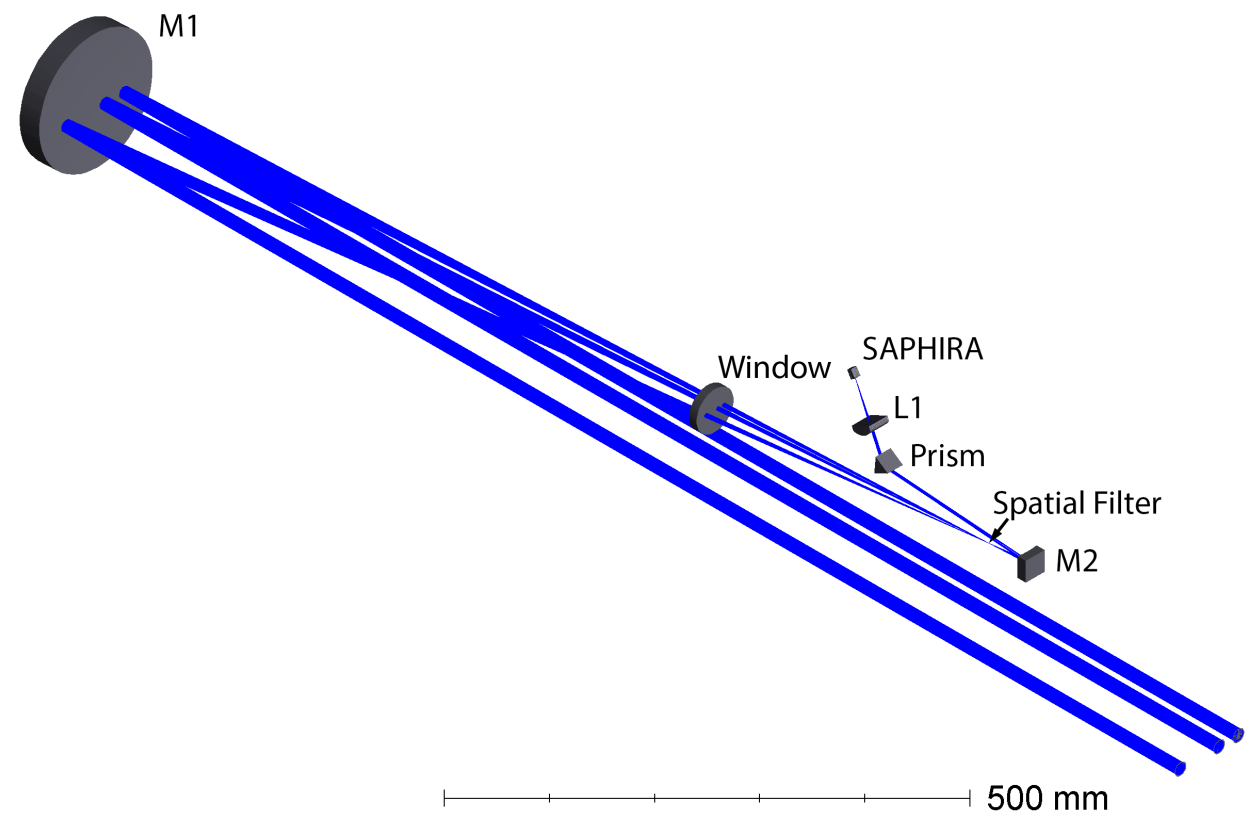

Figure 1. The optical layout of FOURIER. Input beams of diameter $\mathrm{d}=13 \mathrm{~mm}$ are arranged with non-redundant spacings of $2 \mathrm{~d}, 4 \mathrm{~d}$ and $6 \mathrm{~d}$ and travel right to left towards $\mathrm{M} 1$ before being reflected through the cryostat window. They are spatially filtered along the spectral axis by a slit placed at the image plane of M1. The image plane of M2 is then re-imaged onto the SAPHIRA detector ${ }^{6}$ along two perpendicular axes by M2 and L1. The light is spectrally dispersed by the prism between M2 and L1.

Achieving our goal of no motorised mechanics requires FOURIER to be able to access all observing modes for a night's observations without reconfiguration as no operator is allowed within the I-BCA during observations. This was a significant determining factor in our choosing to develop a free space combiner which can pass a wide range of wavelengths (here $1.1 \mu \mathrm{m}-2.4 \mu \mathrm{m}$ ) simultaneously.

\subsubsection{Warm mirror M1}

M1 is a 6 inch diameter gold coated spherical mirror with an average throughput of $98 \%$ in the $\mathrm{J}$ band and $97 \%$ in the $\mathrm{H}$ and $\mathrm{K}$ bands. It serves two functions, the first of which is to re-direct the three beams from the Unit Telescopes (UTs) into the cryostat and towards the cold optics. To reduce the number of optics in FOURIER its second function is to act as the focusing optic for the spatial filtering subsystem. At the image plane of M1 light is spatially filtered by a slit along the spectral dispersion axis. Spatial filtering along the interference fringe axis will be carried out in the data reduction pipeline.

The image plane of M1 must be centred on the spatial filter contained within the cryostat. Given the geometry of the optical design one solution would be to position M1 vertical with the spatial filter along its optical axis. So that the incoming beams do not collide with the cryostat they must be vertically offset from the optical axis of M1. In this configuration M1 would have to have a clear aperture large enough to reflect the incoming beams at their offset landing location. Such a configuration would require a large optic (12 inch diameter for our current 
configuration). An alternative would be to use three independent off-axis parabolas for each beam, however this could lead to misalignments between the beams as they are reflected off independently mounted optics. The solution utilised in FOURIER is a spherical mirror used in an off-axis configuration, where the mirror is tilted and shifted along the surface of the parent sphere until it is centred on the beams, significantly reducing the size of the optic while maintaining a single common path. Another advantage of a monolithic M1 over independent off-axis parabolas is that it allows for the pupil spacing to be changed without the need for any reconfiguration of M1.

\subsubsection{Spatial filter}

Spatial filtering removes higher order spatial perturbations in the beams of starlight induced by atmospheric effects distorting the wavefront. If uncorrected the mismatch in wavefront distortion between two beams being combined from separate telescopes will lead to lower levels of coherence and lower contrast interference fringes. This is a particular problem for beam combiners optimised for sensitivity. While in low light conditions the SNR of an interferometric observation increases linearly with the number of photons, the SNR goes with the square of the fringe contrast. ${ }^{7}$

Two commonly used methods of spatial filtering in image plane beam combination are filtering by single mode fibres, and pinhole aperture filtering. Single mode fibres spatially filter by rejecting all light except that which matches the fundamental mode of the fibre, removing in theory all perturbations to the wavefront and leaving a perfectly spatially filtered beam. Pinhole apertures instead utilise the fact that the image plane is the Fourier transform of the aperture plane and so by passing only the central section of the image plane (typically out to around the first null of the Airy disk) through the pinhole, higher spatial frequency wavefront perturbations are partially removed leaving a spatially filtered beam. Numerical simulations by Keen et $\mathrm{al}^{8}$ show that a pinhole aperture can maintain a visibility as high as $\sim 95 \%$ under seeing conditions as bad as $D / r_{0}=4$ where $D$ is the diameter of the collecting aperture and $r_{0}$ the Fried parameter.

We opted for a pinhole spatial filtering system for FOURIER due to both its simplicity and as it is in keeping with the free space nature of the instrument, allowing us to simultaneously pass $\mathrm{J}, \mathrm{H}$ and $\mathrm{K}$ bands without reconfiguration. An additional benefit to this system is that physical spatial filtering is only required along the spectral axis where the individual Point Spread Functions (PSFs) overlap after dispersion. In the perpendicular interference fringe axis spatial filtering can be carried out in the data reduction pipeline where the width of the digital filter can be optimised for each observation to both the wavelength and atmospheric seeing conditions in a given spectral channel. To take advantage of this, the pinhole in FOURIER is a slit which only filters along the spectral axis.

\subsubsection{Anamorphic pair}

A common issue in image plane beam combination are the two competing requirements of the size of the PSF along the spectral and interference fringe axes. Along the interference axis we wish to sample enough points (typically $\sim 4$ pixels) per cycle of the highest frequency interference fringes formed by the various baselines present. Along the spectral axis however, measuring more samples per spectral channel than is required by Nyquist sampling provides no extra information, and so we wish to minimise the number of pixels read out along this axis to reduce the effects of read noise and to potentially increase the frame rate by using a smaller region of interest on the detector. With the pupil spacing utilised in FOURIER, sampling four pixels/cycle on the highest frequency interference fringes at the shortest wavelength $(1.1 \mu \mathrm{m})$ requires an Airy disk 54 pixels in diameter (to the first dark ring). Satisfying this requirement while only sampling two pixels over the Airy disk along the spectral axis requires an anamorphic factor (the ratio of beam diameter in the interference fringe axis to the spectral axis) of $27 \mathrm{x}$. The resulting PSF for a single beam is shown in figure 2 with the vertical direction being the spectral axis.

The instrument is designed such that the circular PSF at the image plane of M1 must be reshaped into the PSF in figure 2 by magnifying or de-magnifying it along one axis. Placing the requirement of an optical magnification/de-magnification by a factor of $27 \mathrm{x}$ in one axis would be difficult to achieve and likely lead to complex optics which would drive up the cost and technical risk of the project. This high anamorphic factor is instead realised by sharing the magnification/de-magnification between two optics, the anamorphic pair. This 


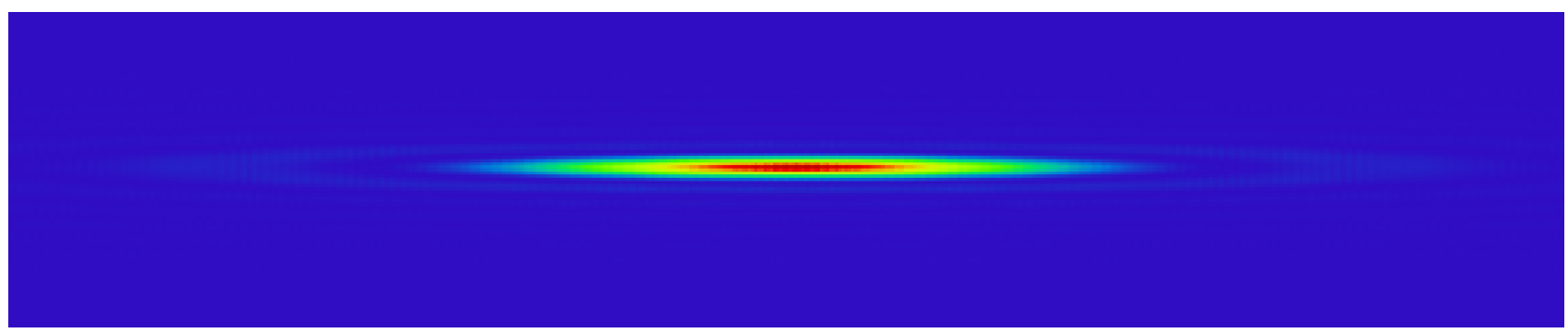

Figure 2. A simulated PSF at $\lambda=1.1 \mu \mathrm{m}$ on the detector plane for the FOURIER instrument with a $27 \mathrm{x}$ anamorphic factor.

was achieved by creating a PSF at the image plane of M1 sized such that a $\sim 5.5 \mathrm{x}$ magnification of it along the interference fringe axis and a $\sim 5.5 \mathrm{x}$ de-magnification of it along the spectral axis gives the desired PSF profile while significantly reducing the magnification/de-magnification requirement on any one optic. In FOURIER this is implemented by a pair of 1 inch cylindrical optics (M2 and L1) with their powered axes perpendicular to each other, M2 and L1 both re-image the focus of M1 onto the SAPHIRA detector.

\subsubsection{Spectral dispersion}

Spectral dispersion in FOURIER is achieved by a simple off-the-shelf equilateral dispersive prism of N-SF11 glass (Thorlabs PS859). This method of dispersion was selected as it is sufficient to reach our design requirement of a spectral resolution of $\mathrm{R}=70$ or above while being easy to implement. The high refractive index of N-SF11 glass $(\mathrm{n}=1.74$ at $\lambda=1.65 \mu \mathrm{m})$ means a high Angle of Incidence (AoI) of $60.9^{\circ}$ for the prism to be operated at its minimum angle of deviation. If left with no Anti Reflection (AR) coating such a high AoI would lead to reflection losses of $13 \%$ across the $\mathrm{J}, \mathrm{H}$ and $\mathrm{K}$ bands at the incident surface of the prism. To maximise throughput the prism will be treated with a custom multi-layer AR coating. A custom coating is necessary due to the unique application of a high AoI, wide spectral coverage and requirement to operate in a liquid nitrogen cooled cryostat. Theoretical curves of the AR coating suggest reflection losses as low as $3.5 \%$ on average at the incident surface of the prism across the $\mathrm{J}, \mathrm{H}$ and $\mathrm{K}$ band are possible.

Another consideration with throughput of N-SF11 glass is the internal transmission which is high in the J and $\mathrm{H}$ bands but drops significantly within the $\mathrm{K}$ band. To minimise these losses the smallest prism usable was selected with $20 \mathrm{~mm}$ faces. As a result, light passing through the centre of the prism passes through $10 \mathrm{~mm}$ of glass, which gives a internal transmission of $99 \%$ at the centres of the $\mathrm{J}$ and $\mathrm{H}$ bands and $90 \%$ at the centre of the $\mathrm{K}$ band.

The benefit of this high dispersion glass is the high spectral resolution achievable with a simple prism. Using the Zemax model of FOURIER to simulate the radius to the first null of the Airy disk along the spectral axis of the PSF, we are able to simulate the separation required to meet the Rayleigh criterion. Comparing the separation of the centroids of the geometric spots at various wavelengths we are then able to estimate the spectral resolution of the system, which is given in table 1 .

Table 1. The spectral resolution of FOURIER at the centres of the J, H and $\mathrm{K}$ bands estimated from the Zemax model by the Rayleigh criterion.

\begin{tabular}{|l|l|l|l|}
\hline & $\mathrm{J}(1.25 \mu \mathrm{m})$ & $\mathrm{H}(1.65 \mu \mathrm{m})$ & $\mathrm{K}(2.2 \mu \mathrm{m})$ \\
\hline$R$ & 90 & 75 & 80 \\
\hline
\end{tabular}

\subsubsection{Throughput}

The optical design of FOURIER is optimised for throughput both by minimising the number of optical surfaces and maximising the throughput of each optical surface. A summary of the expected throughput is given in table 2. Throughput of optical components are taken from Zemax and include the effects of anti-reflection 
coatings and internal transmission losses. The SAPHIRA detector's Quantum Efficiency (QE) is taken from Finger et al. ${ }^{6}$ Atmospheric coupling losses through the spatial filter are estimated from figure 3.17 of Buscher ${ }^{7}$ which models coupling into a single mode fibre for perfectly tip-tilt corrected starlight. The observing conditions were assumed to be $r_{0}=14 \mathrm{~cm}$ at $\lambda=500 \mathrm{~nm}$ (scaled to the $\mathrm{J}, \mathrm{H}$ and $\mathrm{K}$ bands) observed with the $1.4 \mathrm{~m}$ UTs at the MROI.

Table 2. Expected throughput of FOURIER at the centres of the J, H and K bands. Throughput values of optical components are taken from Zemax and include the effects of anti-reflection coatings and internal transmission losses. The SAPHIRA detector's QE is taken from Finger et al. ${ }^{6}$ Atmospheric coupling losses through the spatial filter are estimated from figure 3.17 of Buscher. ${ }^{7}$

\begin{tabular}{|l|l|l|l|}
\hline Component & $\mathrm{J}(1.25 \mu \mathrm{m})$ & $\mathrm{H}(1.65 \mathrm{\mu m})$ & $\mathrm{K}(2.2 \mu \mathrm{m})$ \\
\hline M1 & $98 \%$ & $97 \%$ & $97 \%$ \\
\hline Cryostat window & $95 \%$ & $96 \%$ & $96 \%$ \\
\hline Spatial filter & $34 \%$ & $48 \%$ & $60 \%$ \\
\hline M2 & $98 \%$ & $97 \%$ & $97 \%$ \\
\hline N-SF11 prism & $94 \%$ & $93 \%$ & $86 \%$ \\
\hline L1 & $95 \%$ & $96 \%$ & $96 \%$ \\
\hline SAPHIRA QE & $57 \%$ & $77 \%$ & $84 \%$ \\
\hline \hline Total throughput: & $16 \%$ & $30 \%$ & $38 \%$ \\
\hline
\end{tabular}

Considering the throughput of the optical components alone (without atmospheric coupling losses through the spatial filter and detector QE) the throughput is $81 \%$ in the $\mathrm{J}$ and $\mathrm{H}$ bands and $75 \%$ in the $\mathrm{K}$ band. The only optic from which moderate gains over this high throughput could be made is the N-SF11 prism in the $\mathrm{K}$ band where there is moderate internal absorption. It is also worth noting that while the spatial filter significantly decreases the throughput, the gain in fringe contrast it provides allows us to reach fainter limiting magnitudes than a system with a higher throughput but no spatial filtering. Modelling this throughput alongside all losses associated with the MROI beam train and atmospheric effects, we estimate FOURIER will reach a limiting magnitude of $11.2,11.8$ and 11.4 in the $\mathrm{J}, \mathrm{H}$ and $\mathrm{K}$ bands respectively after five minutes of incoherent integration with exposure times of $2 t_{0}$.

\subsection{Mechanical Design}

\subsubsection{Warm mirror M1}

The functions of M1 are to collect the non-redundantly spaced collimated beams from the UTs and redirect them into the cryostat from the room temperature I-BCA as well as to bring the beams to a focus where they are spatially filtered (see Sec. 2.2.1).

Hence the image plane of M1 must be well aligned with the location of the spatial filter and this places a moderate constraint on the alignment tolerances of M1 to the spatial filter. We have chosen to adjust the image plane of M1 to the spatial filter rather than the spatial filter to the image plane of M1. This approach simplifies the instrument design as M1 is operated at room temperature. The cryostat containing the cold optics can be cooled and then M1 adjusted manually to the location of the spatial filter removing the need for any motorised components, especially within the cryostat. To achieve this three adjusters are placed at the back of M1 allowing for control of tilt and piston. In addition to this the back plate of M1 is tilted at $2.03^{\circ}$ from the vertical, as required to operate $\mathrm{M} 1$ off-axis.

\subsubsection{Spatial filter}

The spatial filter utilised in FOURIER is a slit, acting as a pinhole aperture along the spectral axis while performing no filtering along the interference fringe axis (see Sec. 2.2.2). Numerical simulations of spatial 
filtering via pinholes under atmospheric seeing show that the optimal (maximum SNR) width of a pinhole is slightly less than the width of the first Airy dark ring of the PSF if no atmospheric seeing were present. ${ }^{8}$ This is a challenge for a free space beam combiner such as FOURIER designed to pass the $\mathrm{J}, \mathrm{H}$ and $\mathrm{K}$ bands in the same configuration due to the variation in PSF size as a function of wavelength. Only one wavelength will be optimally spatially filtered, while those longer than it will be spatially filtered more, increasing visibility while reducing throughput. The opposite is true for wavelengths shorter than the optimised wavelength.

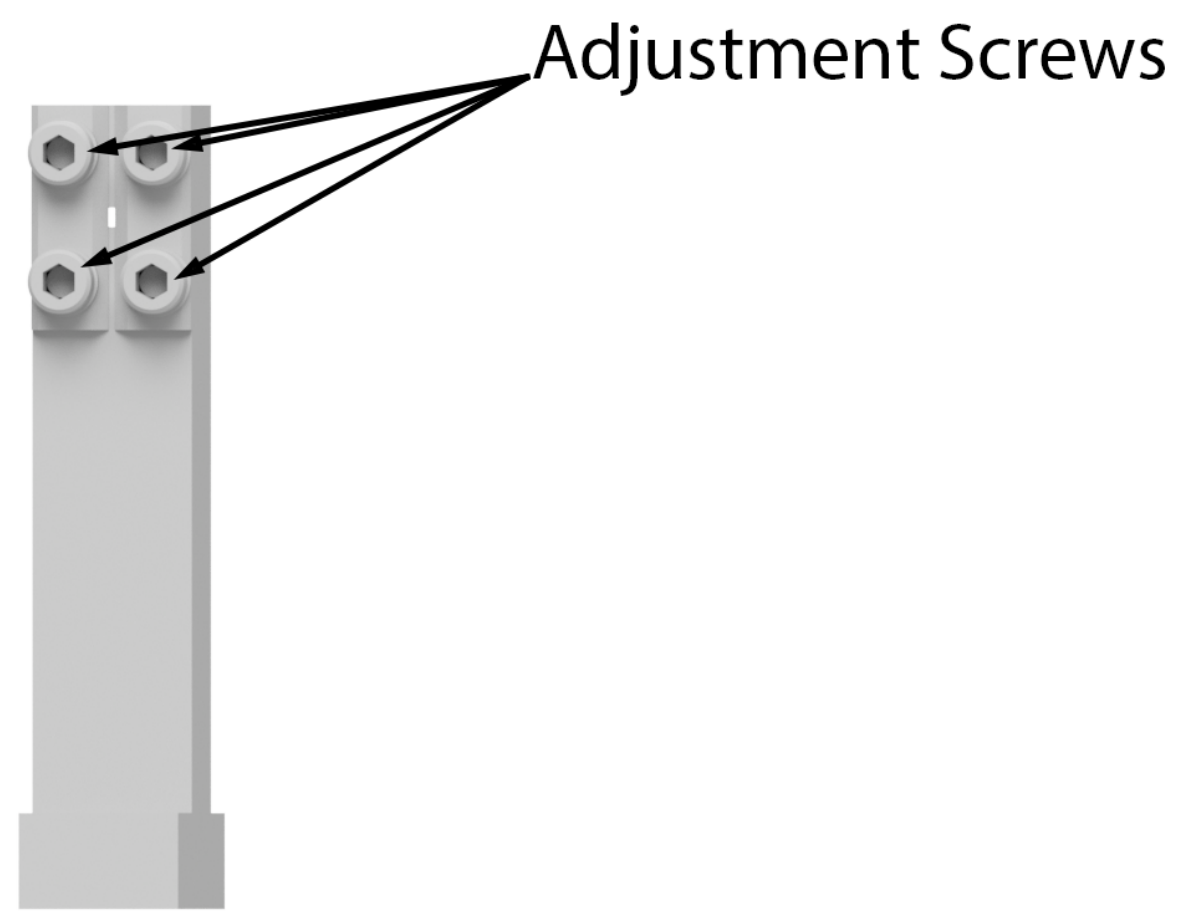

Figure 3. The slit styled spatial filter used in FOURIER. Loosening the four adjustment screws allows the two knife edged plates to move, adjusting the width of the slit.

The current spatial filter in FOURIER will employ a two knife edged plates giving a manually adjustable slit width as shown in figure 3. This is so it can be easily optimised between observing runs for one particular band or optimised as best as it can be for multiple bands. A potential upgrade to FOURIER will be a motorised slit which can be adjusted remotely during observations.

\subsubsection{Cryostat}

A rendering of the FOURIER instrument within the I-BCA at the MROI is shown in figure 4. In keeping with the rest of the instrument the FOURIER cryostat is as simplified as possible. It is entirely passive, containing no pumps (to avoid any induced vibrations) and initially will be manually filled with liquid nitrogen on a daily basis. Two design features in particular have led to an interesting design, the first of which being that M1, the initial focusing optic, reflects the beams back on themselves in the plane of the table (though displaced vertically). As a consequence the cryostat must be placed in the path of the incoming beams so it is raised to allow the incoming beams to pass underneath it. The second is that, given the configuration of the incoming UT beams and M1, the cold optics must be mounted on a vertical cold plate within the cryostat. This cold plate is designed to be removable so the cold optics can be aligned horizontally and later mounted vertically within the cryostat.

\section{LABORATORY TESTS}

Due to a delay in the delivery of the cryostat for the cold optics we are currently conducting room temperature laboratory tests of the instrument to validate the majority of the optical performance criteria under these 


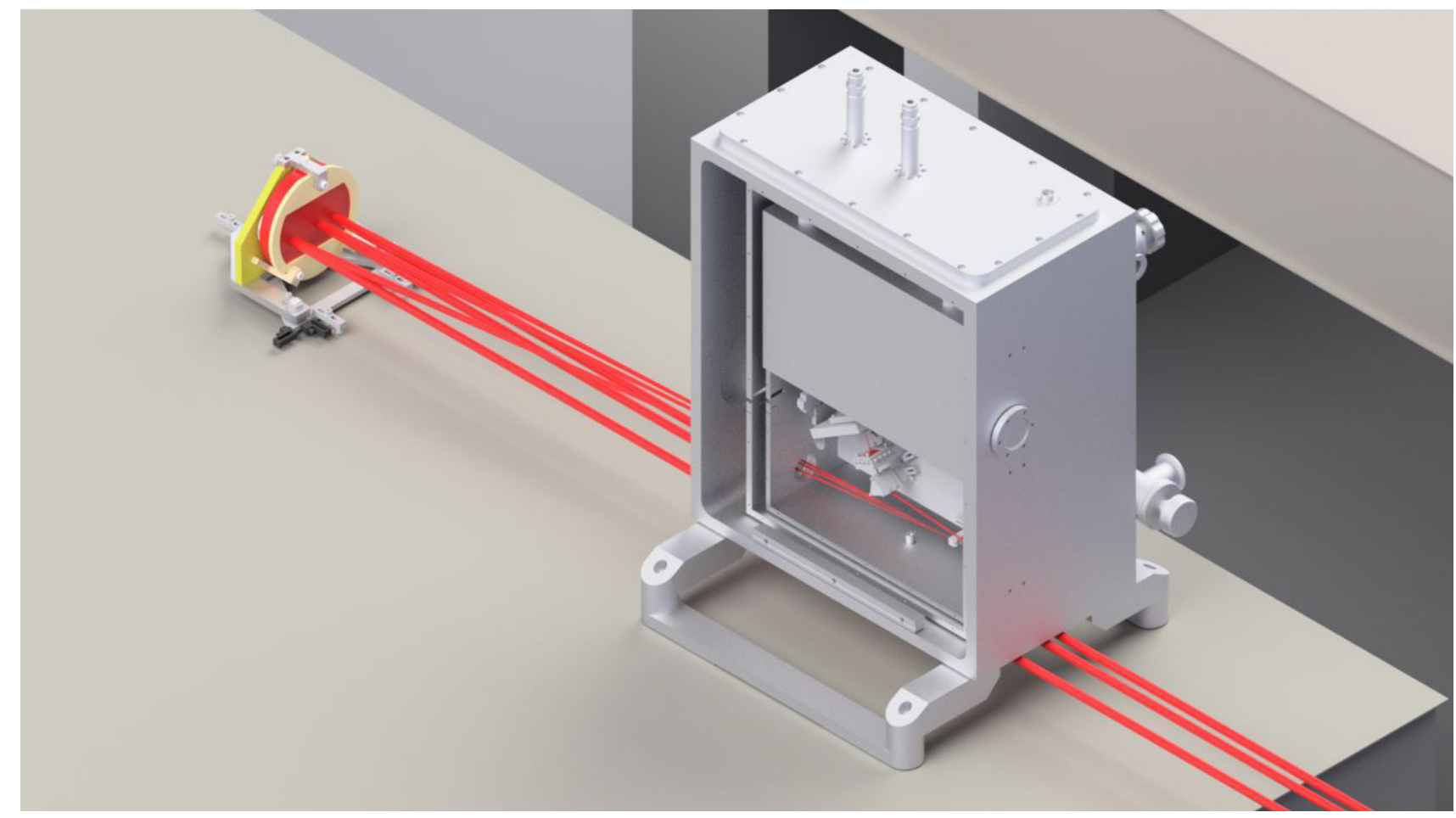

Figure 4. A 3D rendering of the FOURIER instrument in the I-BCA at the MROI array. The three UT beams travel from the bottom right, under the cryostat to the 6 inch spherical mirror M1. They are then reflected into the cryostat. The door, hinges and radiation shields lids have been removed from this render so the beam path within the cryostat can be seen. Note the cold optics mounted vertically beneath the large liquid nitrogen tank.

conditions to enable rapid cold testing at a later stage. The setup for the warm tests is shown in figure 5, here only the spatial filter is missing. We are currently conducting tests on one baseline at a time, the results presented in this paper use two collimated $13 \mathrm{~mm}$ beams separated by $4 \mathrm{x}$ their beam diameters. To enable fine control of path length between the two beams an Agilis AG-LS25-27 linear stage is placed under a periscope (not shown) in the beam path. Another difference to the final version of FOURIER is the camera, here a Raptor Owl 640 Mini is used instead of the final SAPHIRA as the Raptor is designed to operate at room temperature. The pixel pitch of the Raptor is $15 \mu \mathrm{m}$ instead of the $24 \mu \mathrm{m}$ of the SAPHIRA meaning we sample the interference fringes better in the warm laboratory tests. Finally, we are limited in the range of wavelengths we can sample due to the Raptor's cut off wavelength at $\lambda \approx 1.65 \mu \mathrm{m}$. Thus we can only characterise the performance in the $\mathrm{J}$ and $\mathrm{H}$ bands in the current configuration.

\subsection{PSF Shaping}

As discussed in Sec. 2.2.3 the reshaping of the circular PSF is an important feature in the design of FOURIER. Using a $\lambda=1.31 \mu \mathrm{m}$ fibre coupled laser (Thorlabs S3FC1310) as a light source we were able to generate a monochromatic PSF on the FOURIER image plane, the image of which is shown in figure 6 where a cross section through the centre of the PSF along the two perpendicular axes is also plotted. The large ratio of the width of the PSF along the two axes is evident. The width of the first null of the Airy disk is 164 pixels or $2.49 \mathrm{~mm}$ along the interference fringe axis and 6 pixels or $90 \mu \mathrm{m}$ in the spectral axis. This gives a measured anamorphic factor of $27 x$, as expected from the design of FOURIER.

\subsection{Visibility Measurements}

In an ideal system an unresolved point source will generate interference fringes with a visibility of unity. This is not achieved in practise as a number of factors reduce the observed visibility of the target including atmospheric 


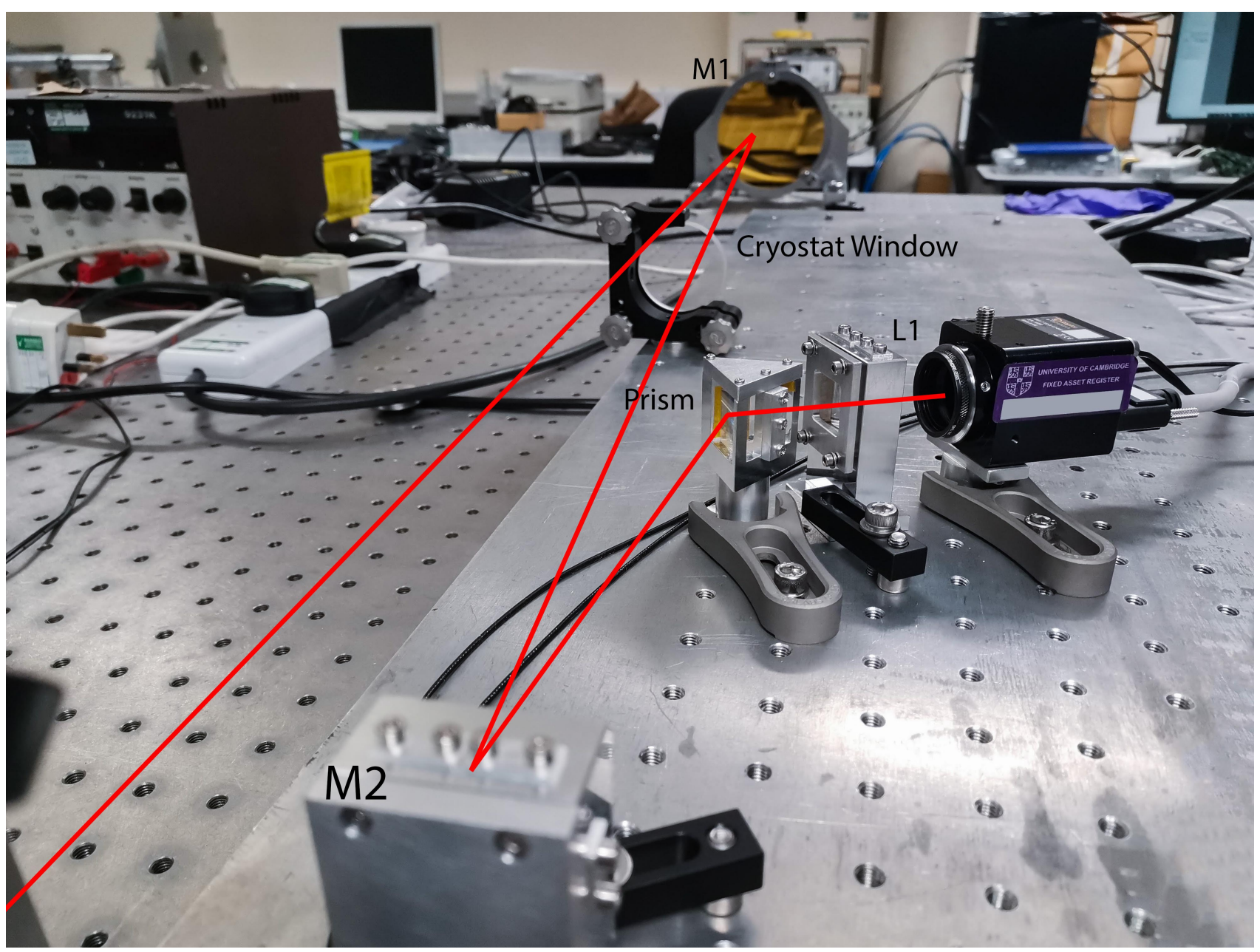

Figure 5. The FOURIER instrument optics in position for warm laboratory tests. Two collimated $13 \mathrm{~mm}$ beams separated by $4 \mathrm{x}$ their beam diameters pass to the left of the cold optics bread board towards M1 where they are reflected through the cryostat window to M2 the prism and L1 before detection on the camera. The optical path is drawn in red. For these warm tests the instrument is rotated $90^{\circ}$ about the axis of the incoming beam compared to its final deployment configuration shown in figure 1.

seeing, finite sampling of the fringe packet and optical aberrations. As the SNR goes with the square of the visibility in low light conditions, maximising it is crucial to building a high sensitivity beam combiner. The purpose of this test then is to quantify any visibility losses and demonstrate that FOURIER is capable of generating high contrast fringes.

To isolate the optical performance of the instrument by minimising other effects which could reduce the fringe contrast (such as polychromatic aberrations in the light source collimation stage), we used the $\lambda=1.31 \mu \mathrm{m}$ fibre coupled laser, collimated by a singlet lens before being split and arranged into two beams directed towards M1. The PSF of the two individual beams and the resulting interference between them is shown in figure 7 where the central lobe and first Airy ring are visible as well as the high anamorphic factor in both beams.

Figure 8 shows the cross section along the interference fringe axis through the centre of the PSFs for both individual beams and the interference fringes. The three cross sections are normalised to the intensity of the fringes profile. We find the two beams are not perfectly overlapped, likely due to a tilt error between the beams in the light source and not FOURIER itself (as all beams through FOURIER share a common path). Beam 2 also has a peak intensity $1.3 \mathrm{x}$ that of Beam 1 which again is likely due to the non-common path which split the 


\section{PSF}

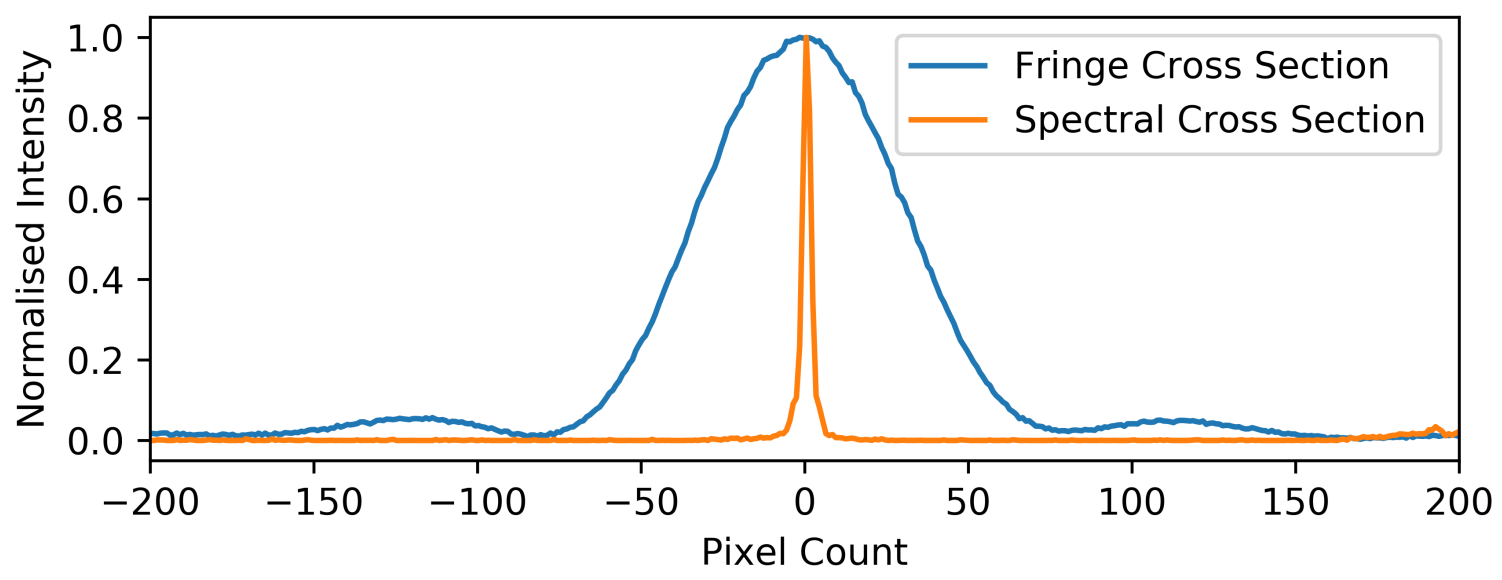

Figure 6. Results of Laboratory measurements. Top: A monochromatic PSF at the FOURIER image plane at $\lambda=1.31 \mu \mathrm{m}$. Bottom: 1D cross sections through the centre of the above PSF along the elongated interference fringe axis and the perpendicular spectral axis. The ratio of the width of the PSF in the two axes (the anamorphic factor) is $27 x$.

laser light and not the common path optics of FOURIER. The relative intensity of the two individual beams to the interference fringes in figure 8 is consistent with the intensity of two superimposed beams at a maximum given by $I_{\text {tot }}=I_{1}+I_{2}+2 \sqrt{I_{1} I_{2}}$.

The modulation transfer function obtained by taking the modulus of the Fourier transform of the fringe cross section in figure 8 gives a measured visibility of $89.2 \%$. The maximum theoretical visibility for two beams with a relative tilt error can be calculated from $V_{\max } \approx 1-(\pi D \theta / \lambda)^{2} / 8$ given by Porro et al. ${ }^{9}$ Where $\theta$ is the tilt between the two beams and $\lambda$ the wavelength. Comparing the shift between centorids of the two PSFs along the interference fringe axis this tilt is estimated to be 4.6 arcseconds which gives a maximum theoretical visibility of $93.8 \%$, or a $6.2 \%$ visibility loss. The visibility loss associated with the difference in intensity of the two beams can be accounted for by calculating the maximum theoretical visibility for the interference of two beams with different intensities by $V_{\max }=2 \sqrt{I_{1} I_{2}} /\left(I_{1}+I_{2}\right)$. Where $I_{1}$ and $I_{2}$ are the intensities of beams one and two respectively. For the $1.3 \mathrm{x}$ difference in intensity between the two measured beams the maximum theoretical visibility of $99.1 \%$, or a $0.9 \%$ loss in visibility. Combining the tilt and mismatched intensity reductions we would expect the maximum observed visibility of our laboratory measurements to be $92.9 \%$. Given this, our measurements contain only a $3.7 \%$ loss in visibility currently unaccounted for. Future laboratory work will address this, particularly attempting to remove the tilt between the two beams from the light source to confirm 


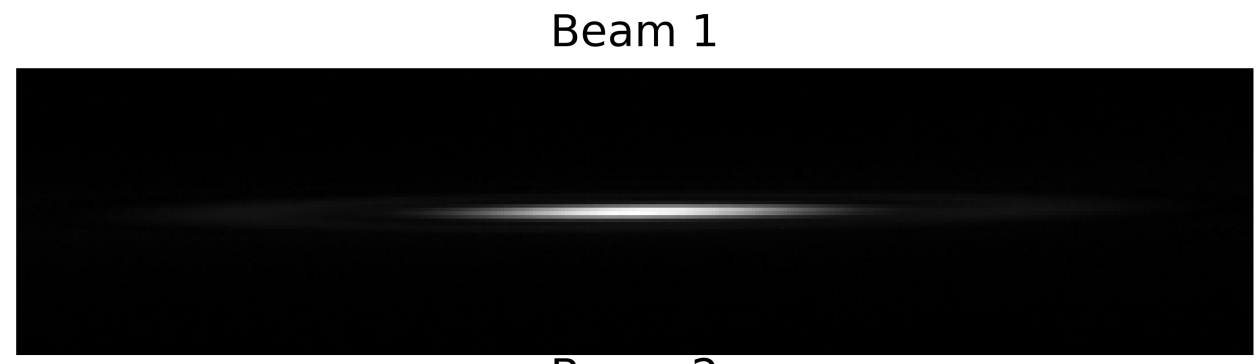

Beam 2

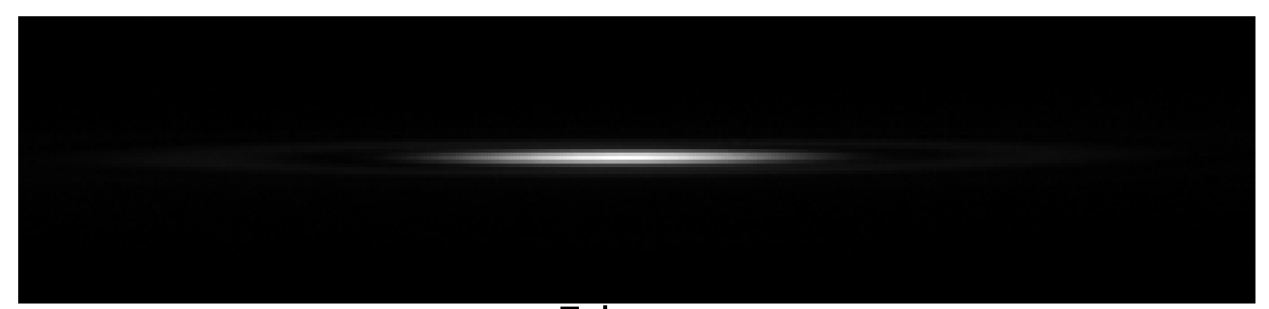

Fringes

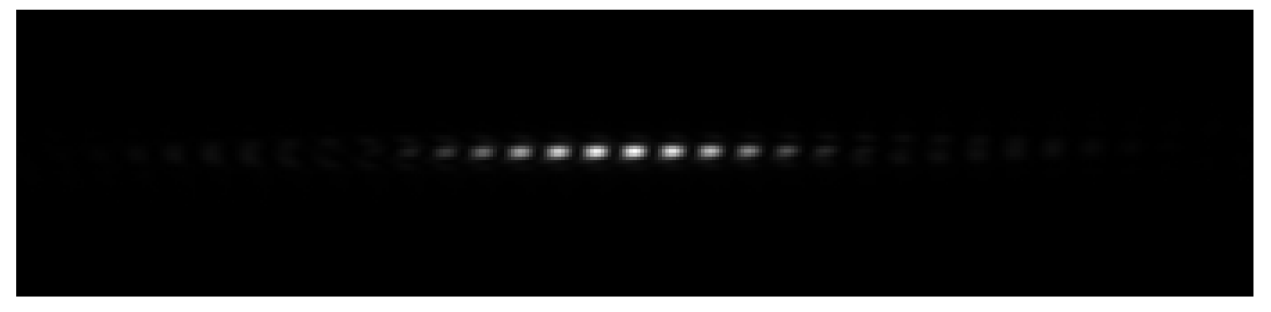

Figure 7. Laboratory measurements of the PSF of the two individual $\lambda=1.31 \mu \mathrm{m}$ laser beams at the image plane of FOURIER and the interference fringes from the resulting superposition of the two. Both the first Airy ring around the central lobe and the high anamorphic factor between the two axes of the PSFs can be seen.

the magnitude of the tilt associated visibility loss.

\subsection{Spectral Resolution}

Using a pinhole filtered collimated white light source, split into two beams separated by $4 \mathrm{x}$ the beam diameter, which were then passed through FOURIER we were able to generate spectrally dispersed interference fringes on the detector. In addition to the white light source the $\lambda=1.31 \mu \mathrm{m}$ fibre coupled laser was propagated along the same path and placed slightly off-axis to separate it from the dispersed white light fringes. The resulting image on the detector is shown in figure 9 with the shorter wavelengths $(\lambda \approx 1.1 \mu \mathrm{m})$ of the dispersed light at the top of the frame, moving to longer wavelengths down the frame until the camera cut-off is reached at $\lambda \approx 1.65 \mu \mathrm{m}$ below which the separated PSF of the $\lambda=1.31 \mu \mathrm{m}$ laser is visible. There is a dip in intensity at $\lambda \approx 1.4 \mu \mathrm{m}$ which is a known characteristic of the light source. Another feature is that the PSF along the interference fringes is significantly larger than our models predict for an ideal system. This is likely due to astigmatism in the light source's spherical collimation mirror being used at an off-axis angle of $17^{\circ}$. Focus was adjusted to achieve best focus along the spectral axis leading to significant aberration in the fringe axis and hence a substantially larger PSF. The $\lambda=1.31 \mathrm{\mu m}$ laser, which is collimated by a different system and does not suffer from the astigmatism, has a much smaller PSF along the interference fringe axis. 


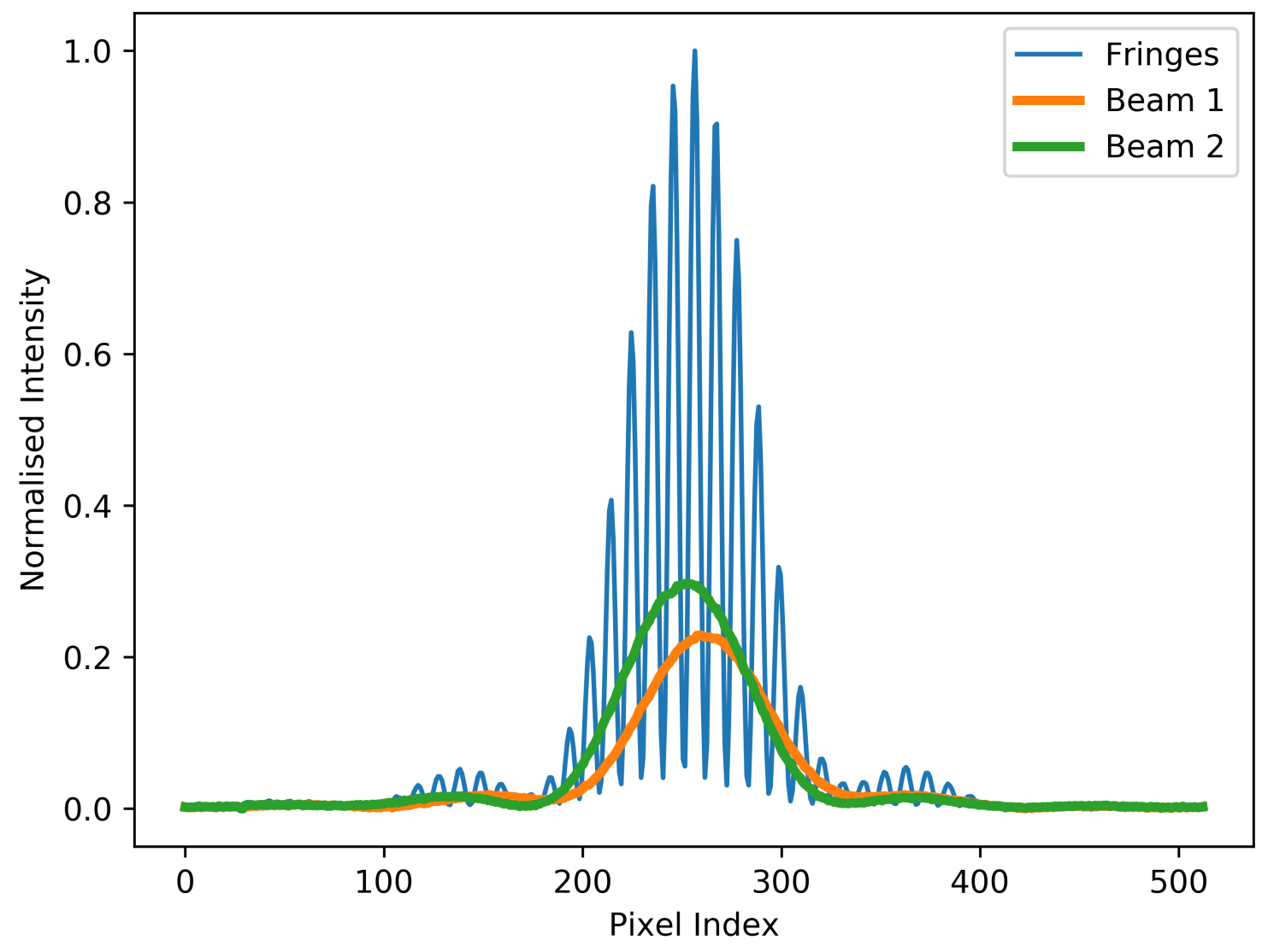

Figure 8. A cross section along the interference fringe axis through the centre of the PSF for the two individual beams and their resulting superposition shown in figure 7. There is a significant error in the overlap of the two individual beams likely due to tilt errors in the light source as the beams experience a common path within FOURIER. The three slices are normalised to the intensity of the fringes and are consistent with the intensities expected for the superposition of two beams.

To quantify the spectral resolution we utilised the configuration shown in figure 9. An Agilis AG-LS25-27 linear stage under the periscope in one of the beams path was used to vary the path length and hence operate the instrument as a Fourier transform spectrometer (FTS). Driving the Agilis at its smallest incremental motion we achieved an average step size of $\sim 100 \mathrm{~nm}$ which, for the configuration tested translated to $\sim 17$ steps of the Agilis per cycle of the fringes at $\lambda=1.65 \mu \mathrm{m}$.

To perform FTS, a frame of the dispersed fringes and laser was taken at each incremental step of the Agilis over the coherence envelope of the dispersed white light fringes. The intensity variation of one pixel in each row of the spectrally dispersed fringes was then extracted from the frames with FTS being carried out on this data. By performing this analysis for every row of pixels we generated an independent spectrum in each row. As the Agilis does not provide information on the distance moved per step, the variation in intensity of the $\lambda=1.31 \mu \mathrm{m}$ laser in a pixel between frames was used to calibrate the change in path length as part of the data reduction.

The spectra recovered from the FTS method for three nearby spectral channels is shown in figure 10. The data is normalised to the maximum intensity in the $\lambda=1.56 \mu \mathrm{m}$ channel though it is not possible to compare channels to estimate the relative throughput as a function of wavelength as the wavelength dependency of the 


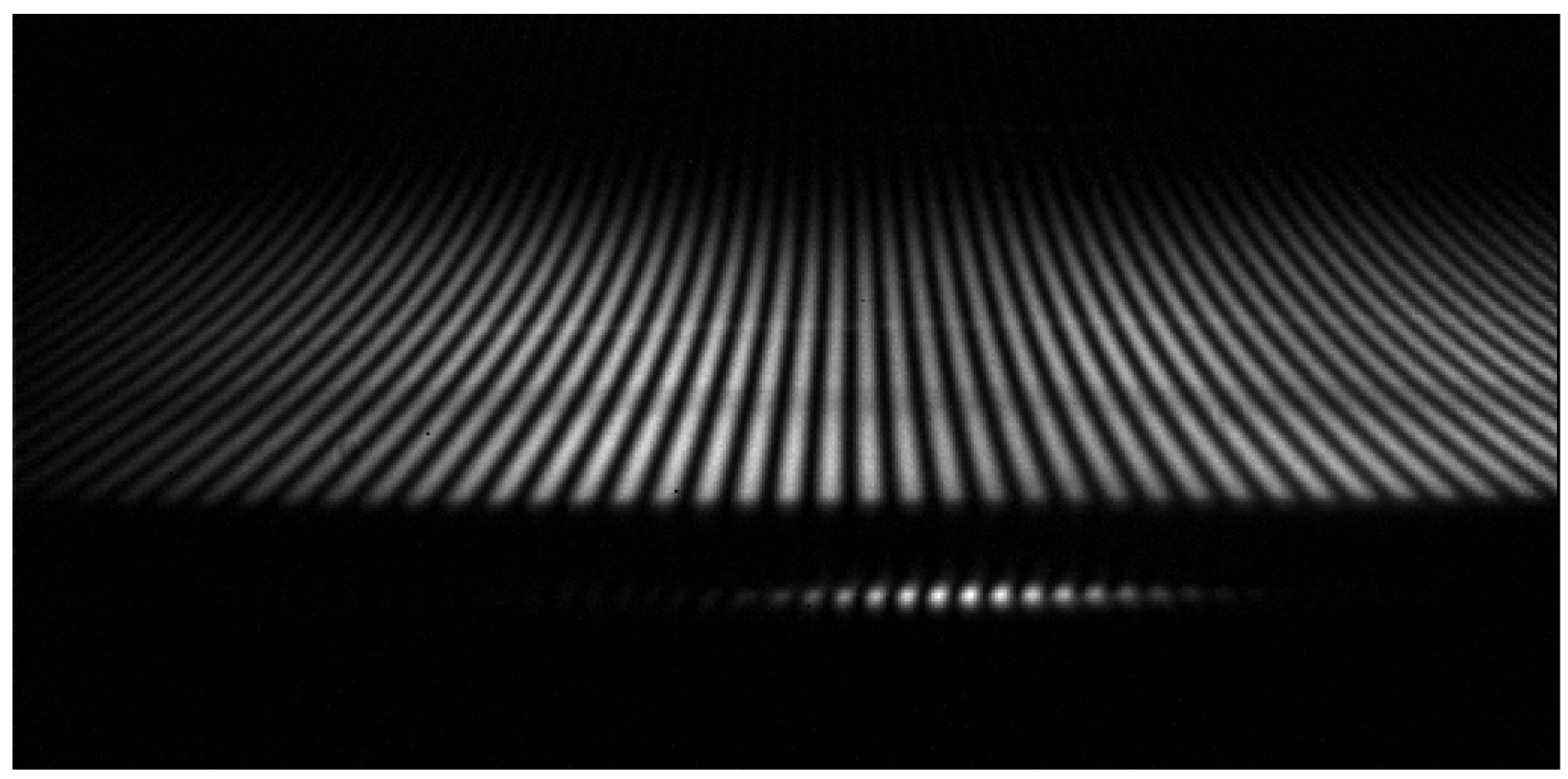

Figure 9. Dispersed interference fringes from $\lambda \approx 1.1 \mu \mathrm{m}-1.65 \mu \mathrm{m}$ (above) and a $\lambda=1.31 \mu \mathrm{m}$ laser (below) at the FOURIER image plane. The dip in intensity at $\lambda \approx 1.4 \mu \mathrm{m}$ is due to a known dip in the light source. The PSF along the interference fringe axis is significantly larger than in the ideal system likely due to astigmatism from an off-axis collimating spherical mirror in the light source.

intensity from the light source has not been factored in. We see a sharp Gaussian-like distribution in all three channels until the intensity drops to roughly $20 \%$ of the peak, at which point a second much broader distribution appears which has not yet been characterised.

Spectral resolution is typically defined by $R=\lambda / \Delta \lambda$ where $\lambda$ is the central wavelength and $\Delta \lambda$ the smallest difference in wavelengths that can be distinguished. Here we define $\Delta \lambda$ as the difference in wavelength across the Full Width at Half Maximum (FWHM) and $\lambda$ as the wavelength at the centre of the Gaussian. To overcome the effects of discrete sampling in the spectra, a Gaussian distribution was fitted to the spectra using the SciPy ${ }^{10}$ curve_fit routine with initial guesses and bounds for the parameters informed by the mean and FWHM of the raw quantised spectra. The wavelengths of the mean and at the FWHM from the best fitting parameters were then used to calculate $R$.

The resulting plot of spectral resolution as a function of wavelength is shown in figure 11, covering wavelengths from the edge of the $\mathrm{J}$ band to the centre of the $\mathrm{H}$ band, beyond which the sensitivity of our room temperature camera cuts off. The values presented here are marginally below the design requirement of $R=70$ in Sec. 2.1 and below the estimated spectral resolution given in Sec. 2.2.4. The reason for this behaviour is currently unknown and will be followed up in future tests.

\section{FUTURE WORK}

The first round of warm laboratory testing is now complete and has shown promising results so far. The immediate next stage will be to conduct more warm laboratory testing to, for example, investigate the visibility loss observed and the throughput of FOURIER, comparing it to the modelled throughput. Once warm tests are completed the next stage of testing will begin in Cambridge where the cold optics will be mounted within the cryostat and the Raptor Owl camera replaced by the SAPHIRA detector. Following the completion of cold tests the instrument will be transported to the MROI array for commissioning. 


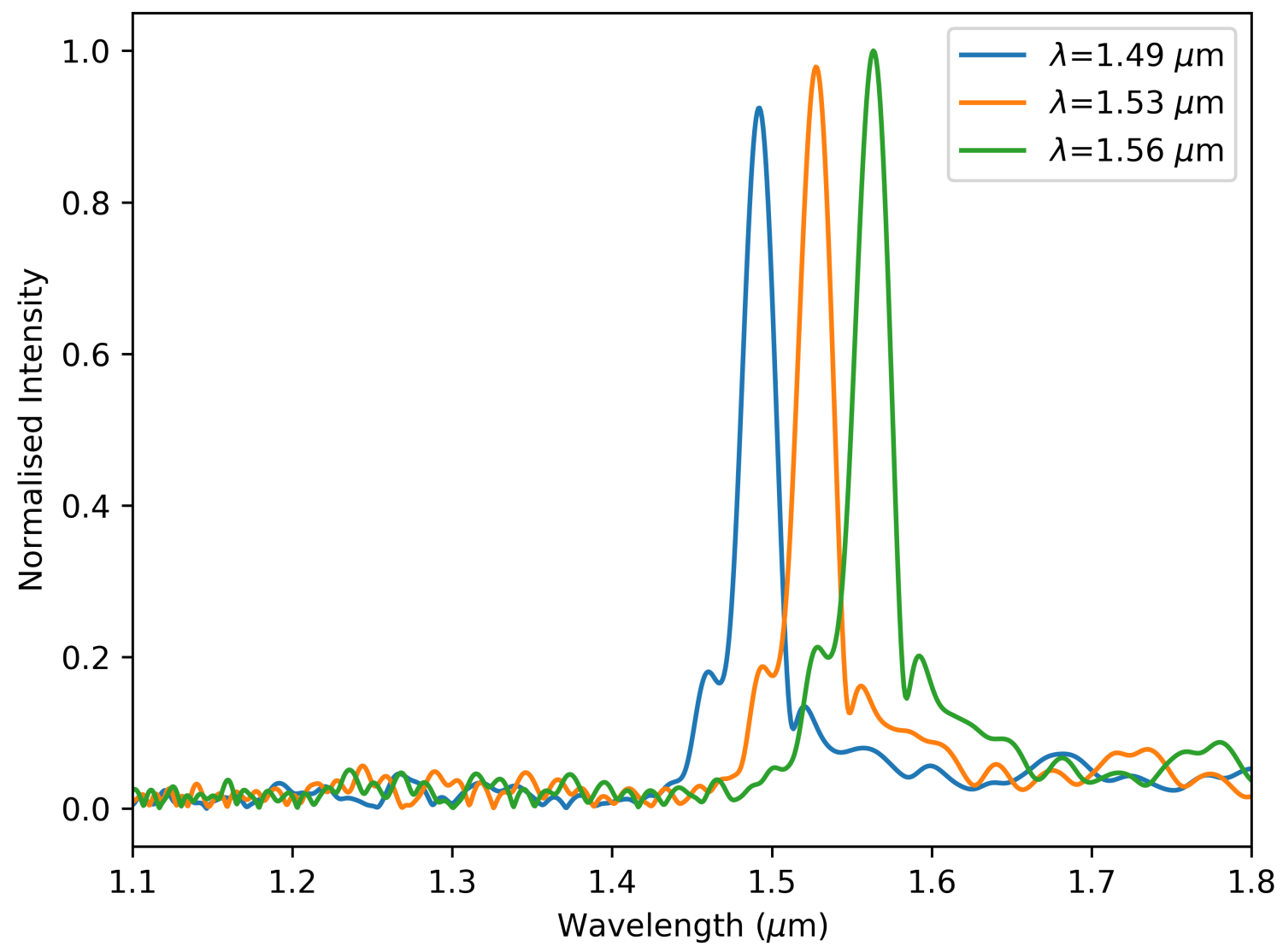

Figure 10. The spectra recovered from the FTS analysis for three channels labelled by the central wavelength of each channel. The three spectra are four rows apart in the spectral direction to roughly match the expected PSF width at these wavelengths on the Raptor camera.

Software associated with FOURIER will be developed over the next year. As FOURIER does not interact in real time with any other subsystem at the MROI or have any motorised mechanics the software requirements are minimal.

\subsection{Cold Tests}

Once we are satisfied that the instrument meets all specifications that can be tested at room temperature we will mount the cold optics within the liquid nitrogen cooled cryostat and replace the Raptor Owl camera with the SAPHIRA detector and perform cold tests. The goals of these cold tests will be to:

- Validate optical alignment can be held to within a tolerable degree during cool-down

- Characterise the temperature gradient across the cold plate within the cryostat and the operating temperature of the SAPHIRA detector

- Test the cryostat requirements such as liquid nitrogen hold time, vacuum levels and stability

- Measure the K band performance 


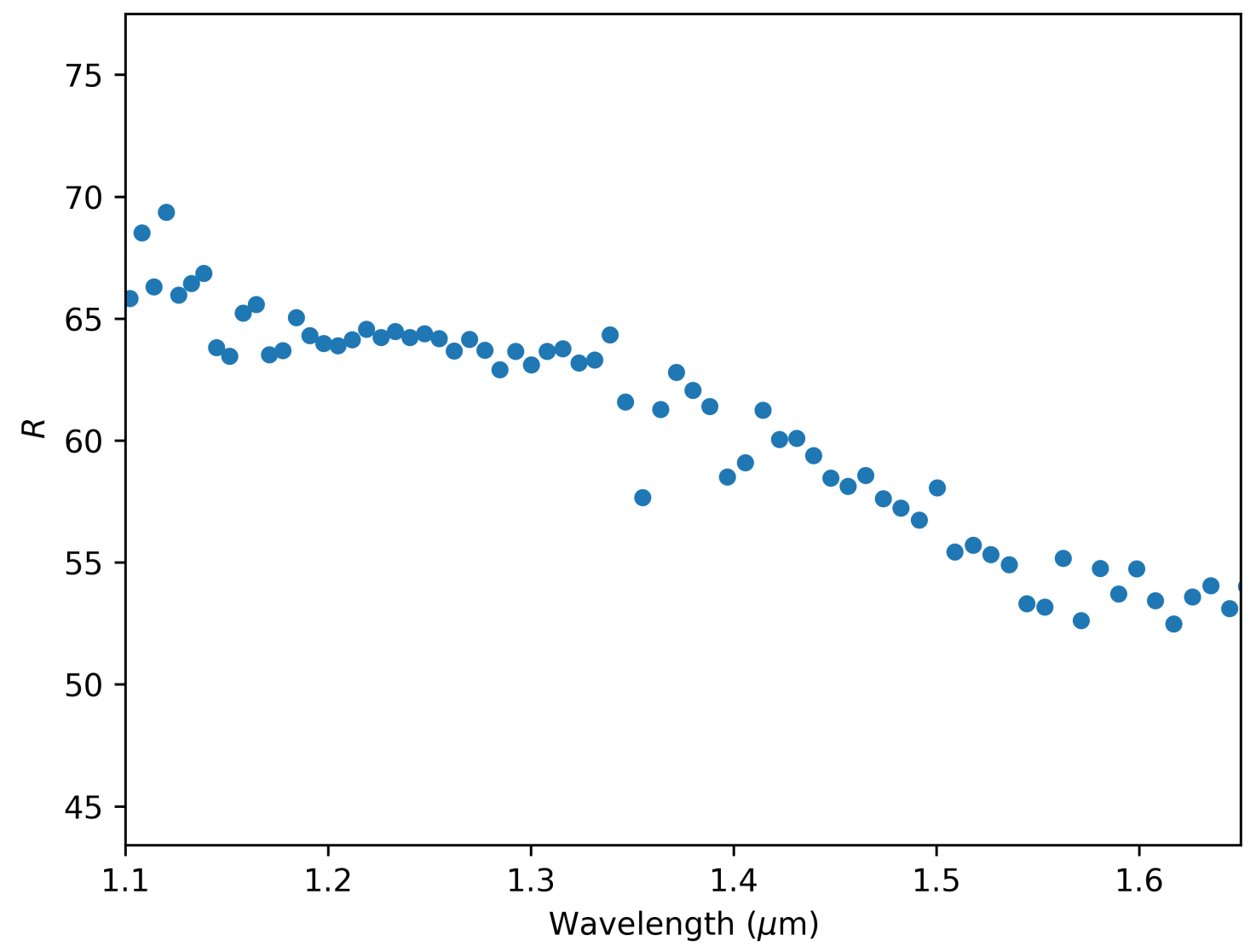

Figure 11. The measured spectral resolution of FOURIER from the edge of the $\mathrm{J}$ band to the centre of the $\mathrm{H}$ band. We are unable to quantify the spectral resolution of wavelengths longer than $\lambda=1.65 \mu \mathrm{m}$ in these tests due to the cut off in sensitivity of our room temperature camera.

- Verify the SAPHIRA low light performance

Cold tests are expected to begin in the second quarter of 2021 in Cambridge in preparation for commissioning work at the MROI.

\subsection{Commissioning at MROI}

Upon completing the cold tests the instrument will be transported from Cambridge, UK to the MROI observatory in New Mexico, USA. Here the instrument will be reassembled in the I-BCA and interfaced with the various subsystems such as the switchyard which will feed the UT beams to FOURIER. Initial tests at the observatory may have access to only one telescope, however, important testing such as validating that star light can be observed from the UT on FOURIER and the quality of that star ligh, as well as measuring the limiting magnitude of a star that can be observed for a single beam can be carried out. With the observing experience on one telescope FOURIER will be ready for observations when the second telescope is online and science verification observations can be carried out immediately. 


\section{CONCLUSIONS}

To support early science at the MROI we have developed FOURIER, a new three way, high sensitivity, J, $\mathrm{H}$ and $\mathrm{K}$ band image plane science beam combiner. Here we have presented the scientific motivation, the optical and mechanical design and results from preliminary warm tests of the instrument. The PSF and its high $27 \mathrm{x}$ anamorphic factor has been measured, visibilities only $3.7 \%$ less than the expected maximum visibility have been measured and an average spectral resolution of $R=62$ has been measured from $\lambda=1.1 \mu \mathrm{m}-1.65 \mu \mathrm{m}$.

The next stages will be to conduct further warm tests and cold tests where the instrument will be configured as it will operate at the MROI. FOURIER will be subsequently commissioned at the MROI in time to conduct the first science observation at the array.

\section{ACKNOWLEDGMENTS}

This material is based on research sponsored by Air Force Research Laboratory (AFRL) under agreement number FA9453-15-2-0086. The U.S. Government is authorized to reproduce and distribute reprints for Governmental purposes notwithstanding any copyright notation thereon. The views and conclusions contained herein are those of the authors and should not be interpreted as necessarily representing the official policies or endorsements, either expressed or implied, of Air Force Research Laboratory (AFRL) and or the U.S. Government.

\section{REFERENCES}

[1] Creech-Eakman, M. J., Romero, V. D., Payne, I., Haniff, C. A., Buscher, D. F., Young, J. S., Santoro, F., Blasi, R., Dahl, C., Dooley, J., Etscorn, D., Farris, A., Fisher, M., Garcia, E., Gino, C., Jaynes, B., Jencka, L., Johnston, P., Jurgenson, C., Kelly, R., Klinglesmith, D., Ligon, E. R., Luis, J., McCracken, T. M., McKeen, C., Mortimer, D., Ochoa, D., Olivares, A., Pino, J., Salcido, C., Schmidt, L. M., Seneta, E. B., Sun, X., and Wilson, D., "The Magdalena Ridge Observatory interferometer: first light and deployment of the first telescope on the array," in [Optical and Infrared Interferometry and Imaging VI], Creech-Eakman, M. J., Tuthill, P. G., and Mérand, A., eds., Society of Photo-Optical Instrumentation Engineers (SPIE) Conference Series 10701, 1070106 (July 2018).

[2] Creech-Eakman, M., Buscher, D. F., Dahl, C., Farris, A., Gino, C., Haniff, C. A., Ligon, E. R., Olivares, A., Payne, I., Romero, V. D., Salcido, C., Santoro, F., and Young, J. S., "The Magdalena Ridge Observatory Interferometer," in [Bulletin of the American Astronomical Society], 51, 131 (Sept. 2019).

[3] Mortimer, D. and Buscher, D., "Prototyping FOURIER: the first generation near-infrared science beam combiner at the MROI," in [Optical and Infrared Interferometry and Imaging VI], Creech-Eakman, M. J., Tuthill, P. G., and Mérand, A., eds., Society of Photo-Optical Instrumentation Engineers (SPIE) Conference Series 10701, 107010C (July 2018).

[4] Weigelt, G., Beckmann, U., Berger, J.-P., Bloecker, T., Brewer, M. K., Hofmann, K.-H., Lacasse, M. G., Malanushenko, V., Millan-Gabet, R., Monnier, J. D., Ohnaka, K., Pedretti, E., Schertl, D., Schloerb, F. P., Scholz, M., Traub, W. A., and Yudin, B., "JHK-band spectro-interferometry of T Cep with the IOTA interferometer," in [Interferometry for Optical Astronomy II], Traub, W. A., ed., Society of Photo-Optical Instrumentation Engineers (SPIE) Conference Series 4838, 181-184 (Feb. 2003).

[5] Buscher, D. F., Creech-Eakman, M., Farris, A., Haniff, C. A., and Young, J. S., "The Conceptual Design of the Magdalena Ridge Observatory Interferometer," Journal of Astronomical Instrumentation 2, 1340001 (Dec. 2013).

[6] Finger, G., Baker, I., Alvarez, D., Dupuy, C., Ives, D., Meyer, M., Mehrgan, L., Stegmeier, J., and Weller, H. J., "Sub-electron read noise and millisecond full-frame readout with the near infrared eAPD array SAPHIRA," in [Adaptive Optics Systems V], Marchetti, E., Close, L. M., and Véran, J.-P., eds., Society of Photo-Optical Instrumentation Engineers (SPIE) Conference Series 9909, 990912 (July 2016).

[7] Buscher, D. F., [Practical Optical Interferometry], Cambridge University Press, Cambridge (2015).

[8] Keen, J. W., Buscher, D. F., and Warner, P. J., "Numerical simulations of pinhole and single-mode fibre spatial filters for optical interferometers," 326, 1381-1386 (Oct. 2001).

[9] Porro, I. L., Traub, W. A., and Carleton, N. P., "Effect of Telescope Alignment on a Stellar Interferometer," 38, 6055-6067 (Oct. 1999). 
[10] Virtanen, P., Gommers, R., Oliphant, T. E., Haberland, M., Reddy, T., Cournapeau, D., Burovski, E., Peterson, P., Weckesser, W., Bright, J., van der Walt, S. J., Brett, M., Wilson, J., Millman, K. J., Mayorov, N., Nelson, A. R. J., Jones, E., Kern, R., Larson, E., Carey, C. J., Polat, I., Feng, Y., Moore, E. W., VanderPlas, J., Laxalde, D., Perktold, J., Cimrman, R., Henriksen, I., Quintero, E. A., Harris, C. R., Archibald, A. M., Ribeiro, A. H., Pedregosa, F., van Mulbregt, P., and SciPy 1.0 Contributors, "SciPy 1.0: Fundamental Algorithms for Scientific Computing in Python," Nature Methods 17, 261-272 (2020). 